COMMENTARY

\title{
Amiodarone-induced thyrotoxicosis: something new to refine the initial diagnosis?
}

\author{
Maria Laura Tanda, Fausto Bogazzi ${ }^{1}$, Enio Martino ${ }^{1}$ and Luigi Bartalena \\ Division of Endocrinology, Department of Clinical Medicine, University of Insubria, Ospedale di Circolo, Viale Borri, 57, 21100 Varese, Italy and \\ ${ }^{1}$ Department of Endocrinology and Metabolism, University of Pisa, 56124 Pisa, Italy \\ (Correspondence should be addressed to L Bartalena; Email: l.bartalena@libero.it; luigi.bartalena@uninsubria.it)
}

Amiodarone-induced thyrotoxicosis (AIT) is a clinical condition fraught with difficulties and uncertainties both from the diagnostic and therapeutic standpoints (1). Diagnosis and treatment are closely linked, because the diagnostic problems may affect the therapeutic approach and lead to suboptimal treatment and delayed resolution of dysfunction. This, in turn, is risky for the AIT patient, who generally has underlying heart abnormalities (arrhythmias, heart failure) for which prompt restoration and stable maintenance of euthyroidism represent fundamental actions (1). Indeed, AIT is associated with increased mortality, particularly in elderly patients with markedly impaired left ventricular function (2). Thus, at variance with amiodaroneinduced hypothyroidism (3), AIT poses relevant and urgent therapeutic problems.

What is difficult in AIT is not to diagnose the thyrotoxic state, but the pathophysiologic mechanism(s) underpinning it. Traditionally, although schematically, two main forms of AIT are distinguished (4). Type 1 AIT is a classical form of iodine-induced thyrotoxicosis associated with true hyperthyroidism; thyroid hyperfunction is triggered by the iodine load, and the thyroid gland generally is intrinsically abnormal owing to latent autonomy due to thyroid nodular or autoimmune disease. Type 2 AIT is a form of destructive thyroiditis caused by iodine or, more likely, amiodarone or its metabolite, desethylamiodarone; most cases develop in normal thyroid glands, although a small, diffuse or nodular goiter may sometimes be present. AIT may occur at any time during amiodarone therapy and even long after amiodarone withdrawal (1). Clinical presentation of the two forms does not differ (1). A relevant proportion (about 20\%) of patients with type 2 AIT develop hypothyroidism in the long run (5). Type 1 AIT is treated with thionamides with or without potassium perchlorate, but it often is resistant to therapy because of the large intrathyroidal iodine stores (1); on the contrary, type 2 AIT is managed with oral glucocorticoids (6), but the time required to normalize thyroid status may vary depending mainly on thyroid volume and severity of baseline thyrotoxicosis (7).

Type 2 is nowadays the predominant form of AIT. In a review of our own cases, over a 27 -year period, type 1 AIT was the most frequent form (about $60 \%$ of cases) during the first years covered by the study, but type 2
AIT more recently accounted for $\sim 90 \%$ of cases (8). The reasons for this change in proportion are unclear; however, it is conceivable that patients who are candidates for elective amiodarone treatment are nowadays screened for pre-existing thyroid abnormalities more frequently than in the past prior to amiodarone administration. In addition, availability of other therapeutic procedures and effective anti-arrhythmic drugs may account for a more limited use of amiodarone in patients with underlying subclinical thyroid disease.

As we alluded to, the above classification does not take into account the large grey zone of mixed (or indefinite) forms of AIT whereby both pathogenic mechanisms - excessive thyroid hormone synthesis and thyroid destructive phenomena - coexist and concur to thyrotoxicosis. Treatment of these forms is based on the concomitant use of thionamides (with or without potassium perchlorate) and glucocorticoids. The diagnostic uncertainty surrounding these forms of AIT is well reflected by recent questionnaire-based surveys carried out among expert thyroidologist members of the European Thyroid Association (9), Latin American Thyroid Society (10), and American Thyroid Association (11). In general, measurement of thyroidal radioactive iodine uptake (RAIU) and assessment by color flow Doppler sonography (CFDS) are considered the best tools to differentiate type 1 and type 2 AIT (12-14). In fact, RAIU usually is very lowto-suppressed in type 2 AIT and low-to-normal in type 1 AIT, while CFDS usually show a pattern 0 (absent vascularity) in type 2 AIT and a pattern I to III (normal-to-increased vascularity) in type 1 AIT. Other parameters, such as serum interleukin (IL)6, C-reactive protein, and thyroglobulin are considered to be of controversial or no usefulness in the differential diagnosis (14-17). Despite the combination of RAIU and CFDS in the diagnostic work up of an ideal AIT patient presented in the mentioned questionnaire, about $15 \%$ of American respondents and 27\% of European respondents were unable to make a clear-cut diagnosis of type 1 or type 2 AIT and propended for a diagnosis of mixed (or indefinite) form of AIT (11).

Can we refine and improve the diagnostic criteria of AIT? The paper by Piga et al. (18) in this issue of the European Journal of Endocrinology is interesting in this 
regard. The authors evaluated a series of 20 consecutive AIT patients, who were initially classified, according to standard criteria, as having type 1 AIT $(n=8)$ or type 2 AIT $(n=12)$. The final diagnosis was also based on the response to different treatments for the thyrotoxicosis. Two patients initially treated with methimazole and potassium perchlorate following a diagnosis of type 1 AIT, required the addition of glucocorticoids to restore euthyroidism; conversely, two patients, initially treated with glucocorticoids after a diagnosis of type 2 AIT, needed the addition of methimazole and potassium perchlorate to normalize their metabolic status. These four patients, according to the authors, met a posteriori criteria for the diagnosis of a mixed form of AIT. All patients had been submitted to thyroid ${ }^{99 \mathrm{~m}} \mathrm{Tc}$ 2-methoxy-isobutyl-isonitrile (MIBI) scintigraphy before any treatment was initiated. Assessment of MIBI scan results showed that MIBI diffuse retention, suggestive of hyperfunctioning tissue, was present in all six patients with a final diagnosis of type 1 AIT, while no significant uptake, expression of a necrotic process, was found in the 10 patients with a final diagnosis of type 2 AIT. Of the four patients whose final diagnosis, based on therapeutic outcome, was mixed (or indefinite) AIT, two had a faint persistent MIBI uptake, and two a rapid washout of the tracer. Interestingly, some type 1 AIT patients had very low RAIU values, and three mixed AIT patients had CFDS features suggestive of type 2 AIT. Thus, Piga et al. (18) suggest that MIBI may represent a useful tool in the differential diagnosis of different forms of AIT, particularly for the identification of mixed forms, the most resistant to medical treatment.

Some caution is warranted in the interpretation of these results. A very important limitation of this study is the small number of enrolled patients. Only six patients with type 1 AIT were included. The latter is the most critical category of patients, because it is more likely to find patients with mixed forms of AIT among this subgroup than among patients with clear-cut destructive phenomena, although also two patients initially classified as having type 2 AIT eventually proved to have a mixed form of AIT. A second important point is the following. MIBI was positive in two patients with an initial diagnosis of type 2 AIT, who subsequently required the addition of methimazole and potassium perchlorate because glucocorticoids alone were unsuccessful. In these two patients, MIBI positivity, together with other features compatible with type 2 AIT, might have suggested an initial diagnosis of a mixed form of AIT and, therefore, the combination of the three drugs from the very beginning. However, two patients with an initial diagnosis of type 1 AIT and positive MIBI scan then required the addition of glucocorticoids to antithyroid drugs to restore euthyroidism; in these two patients ( $25 \%$ of those initially diagnosed type 1 AIT), MIBI was not a relevant tool to diagnose a mixed form of AIT and would probably not have changed the initial therapeutic approach based on the use of antithyroid drugs without glucocorticoids. Thus, the advantage provided by MIBI scan in the initial diagnosis seems to be greater for those mixed forms apparently falling into the category of pure type 2 AIT than for those apparently falling into the category of pure type 1 AIT. Although, as mentioned above, type 2 AIT is by far the most prevalent form of AIT, this limitation of MIBI in differentiating type 1 AIT from mixed forms should be considered. In type 2 AIT, MIBI is probably useful to decide the most correct, multipharmacologic treatment after the initial diagnosis without any further waste of time. It seems appropriate to propose that a large multicenter study should be undertaken to enroll a sufficiently high number of patients to confirm these preliminary results.

\section{References}

1 Martino E, Bartalena L, Bogazzi F \& Braverman LE. The effects of amiodarone on the thyroid. Endocrine Reviews 200122 240-254.

2 O'Sullivan AJ, Lewis M \& Diamond T. Amiodarone-induced thyrotoxicosis: left ventricular dysfunction is associated with increased mortality. European Journal of Endocrinology 2006154 $533-536$

3 Bogazzi F, Bartalena L, Tomisti L, Dell'Unto E, Cosci C, Sardella C, Tanda ML, Lai A, Gasperi M, Aghini-Lombardi F \& Martino E. Potassium perchlorate only temporarily restores euthyroidism in patients with amiodarone-induced hypothyroidism who continue amiodarone therapy. Journal of Endocrinological Investigation 2008 31 515-519.

4 Bogazzi F, Bartalena L, Gasperi M, Braverman LE \& Martino E. The various effects of amiodarone on thyroid function. Thyroid 2001 11 511-519.

5 Bogazzi F, Dell'Unto E, Tanda ML, Tomisti L, Cosci C, AghiniLombardi F, Sardella C, Pinchera A, Bartalena L \& Martino E. Long-term outcome of thyroid function after amiodarone-induced thyrotoxicosis, as compared to subacute thyroiditis. Journal of Endocrinological Investigation 200729 694-699.

6 Bartalena L, Brogioni S, Grasso L, Bogazzi F, Burelli A \& Martino E. Treatment of amiodarone-induced thyrotoxicosis, a difficult challenge: results of a prospective study. Journal of Clinical Endocrinology and Metabolism 199681 2930-2933.

7 Bogazzi F, Bartalena L, Tomisti L, Rossi G, Tanda ML, Dell'Unto E, Aghini-Lombardi F \& Martino E. Glucocorticoid response in amiodarone-induced thyrotoxicosis resulting from destructive thyroiditis is predicted by thyroid volume and serum free thyroid hormone concentrations. Journal of Clinical Endocrinology and Metabolism 200792 556-562.

8 Bogazzi F, Bartalena L, Dell'Unto E, Tomisti L, Rossi G, Pepe P, Tanda ML, Grasso L, Macchia E, Aghini-Lombardi F, Pinchera A \& Martino E. Proportion of type 1 and type 2 amiodarone-induced thyrotoxicosis has changed over a 27-year period in Italy. Clinical Endocrinology 200767 533-537.

9 Bartalena L, Wiersinga WM, Tanda ML, Bogazzi F, Piantanida E, Lai A \& Martino E. Diagnosis and management of amiodaroneinduced thyrotoxicosis in Europe: results of an international survey among members of the European Thyroid Association. Clinical Endocrinology 200461 494-502.

10 Diehl LA, Romaldini JH, Graf H, Bartalena L, Martino E, Albino CC \& Wiersinga WM. Management of amiodarone-induced thyrotoxicosis in Latin America: an electronic survey. Clinical Endocrinology 200665 433-438.

11 Tanda ML, Piantanida E, Lai A, Liparulo G, Sassi L, Bogazzi F, Wiersinga WM, Braverman LE, Martino E \& Bartalena L. 
Diagnosis and management of amiodarone-induced thyrotoxicosis: similarities and differences between North Americans and European thyroidologists. Clinical Endocrinology, 2008. Epub ahead of print.

12 Bogazzi F, Martino E, Dell'Unto E, Brogioni S, Cosci C, AghiniLombardi F, Ceccarelli C, Pinchera A, Bartalena L \& Braverman LE. Thyroid color flow Doppler sonography and radioiodine uptake in 55 consecutive patients with amiodarone-induced thyrotoxicosis. Journal of Endocrinological Investigation 200326 635-640.

13 Loy M, Perra E, Melis A, Cianchetti ME, Piga M, Serra A, Pinna G \& Mariotti S. Color-flow Doppler sonography in the differential diagnosis and management of amiodarone-induced thyrotoxicosis. Acta Radiologica 200748 628-634.

14 Bartalena L, Brogioni S, Grasso L, Rago T, Vitti P \& Martino E. Interleukin-6: a marker of thyroid-destructive processes? Journal of Clinical Endocrinology and Metabolism 199479 1424-1427.

15 Daniels GH. Amiodarone-induced thyrotoxicosis. Journal of Clinical Endocrinology and Metabolism 200186 3-8.
16 Eaton SEM, Euinton HA, Newman CM, Weetman AP \& Bennet WM. Clinical experience of amiodarone-induced thyrotoxicosis over a 3-year period: role of colour-flow Doppler sonography. Clinical Endocrinology 200256 33-38.

17 Pearce EN, Bogazzi F, Martino E, Brogioni S, Pardini E, Pellegrini G, Parkes AB, Lazarus JH, Pinchera A \& Braverman LE. The prevalence of elevated serum C-reactive protein levels in inflammatory and noninflammatory thyroid disease. Thyroid 200313 643-648.

18 Piga M, Cocco MC, Serra A, Loy M, Boi F \& Mariotti S. The usefulness of ${ }^{99 \mathrm{~m}} \mathrm{Tc}$-sestaMIBI thyroid scan in the differential diagnosis and management of amiodarone-induced thyrotoxicosis. European Journal of Endocrinology $2008159423-429$.

Received 25 July 2008

Accepted 29 July 2008 